\title{
The extracellular matrix proteoglycan perlecan facilitates transmembrane semaphorin-mediated repulsive guidance
}

\author{
Joong Youn Cho, Kayam Chak, Benjamin J. Andreone, Joseph R. Wooley, and Alex L. Kolodkin ${ }^{1}$ \\ The Solomon H. Snyder Department of Neuroscience, Howard Hughes Medical Institute, The Johns Hopkins University \\ School of Medicine, Baltimore, Maryland 21205, USA
}

The Drosophila transmembrane semaphorin-1a (Sema-1a) is a repulsive guidance cue that uses the Plexin A (PlexA) receptor during neural development. Sema-1a is required in axons to facilitate motor axon defasciculation at guidance choice points. We found that mutations in the trol gene strongly suppress Sema-1a-mediated repulsive axon guidance. trol encodes the phylogenetically conserved secreted heparan sulfate proteoglycan (HSPG) perlecan, a component of the extracellular matrix. Motor axon guidance defects in perlecan mutants resemble those observed in Sema-1a- and PlexA-null mutant embryos, and perlecan mutants genetically interact with $\operatorname{Plex} A$ and Sema-1a. Perlecan protein is found in both the CNS and the periphery, with higher expression levels in close proximity to motor axon trajectories and pathway choice points. Restoring perlecan to mutant motor neurons rescues perlecan axon guidance defects. Perlecan augments the reduction in phospho-focal adhesion kinase (phospho-FAK) levels that result from treating insect cells in vitro with Sema-1a, and genetic interactions among integrin, Sema-1a, and $F A K$ in vivo support an antagonistic relationship between Sema-1a and integrin signaling. Therefore, perlecan is required for Sema-1a-PlexA-mediated repulsive guidance, revealing roles for extracellular matrix proteoglycans in modulating transmembrane guidance cue signaling during neural development.

[Keywords: axon guidance; semaphorin; perlecan; Drosophila; extracellular matrix; proteoglycan]

Supplemental material is available for this article.

Received April 2, 2012; revised version accepted August 1, 2012.

Correct nervous system function depends on precise, yet complex, connections among neuronal processes and their targets (Tessier-Lavigne and Goodman 1996). Transmembrane and secreted guidance cues, working as either attractants or repellents, guide extending axons as they establish their trajectories. Although several families of guidance cues have been characterized (Kolodkin and Tessier-Lavigne 2011), it is still unclear how the complexity of neural connectivity arises through the action of a limited number of cues. One way is through modulation of guidance cue signaling by extrinsic factors and also intracellular signaling components (Van Vactor et al. 2006; Bashaw and Klein 2010), although precisely how this modulation occurs is only incompletely understood. Drosophila embryonic neural development provides an excellent model system to identify these modulators and determine how they function during the establishment of neuronal connectivity.

The extracellular environment, where axons and dendrites encounter guidance cues, contains a myriad of ex-

${ }^{1}$ Corresponding author

E-mail kolodkin@jhmi.edu

Article is online at http://www.genesdev.org/cgi/doi/10.1101/gad.193136.112. tracellular matrix (ECM) components with the potential to influence guidance cue function (Van Vactor et al. 2006). Detailed anatomical studies show that growth cones make direct contact with the ECM in vivo (Roberts and Taylor 1982), and ECM ligands and their receptors, such as laminins and integrins, respectively, regulate axon pathfinding in vivo (García-Alonso et al. 1996; Hoang and Chiba 1998; Myers et al. 2011).

Guidance cue receptors are distributed both uniformly and in a compartmentalized fashion along navigating axons (Katsuki et al. 2009; Winckler and Mellman 2010). However, it is at specific choice points where steering responses to guidance cues must occur in order for precise axon trajectories to be established (Raper and Mason 2010). The distribution of ECM components at select locations and at specific times during development can provide such information for secreted guidance cues. However, modulation of neuronal transmembrane guidance cue ligandreceptor interactions in vivo by ECM components remains to be investigated.

Proteoglycans consist of a core protein that is covalently modified with different classes of glycosaminoglycan (GAG) side chains: heparan sulfate (HS) and chondroitin sulfate (CS) (Van Vactor et al. 2006). Although initially 
considered as only structural ECM components, proteoglycans are now known as modulators of ligand-receptor signaling events in numerous developmental contexts (Van Vactor et al. 2006). Analysis of mice lacking an HS chain biosynthetic enzyme in the nervous system demonstrates that HSs play an essential role in axon guidance at the CNS midline (Inatani et al. 2003). Importantly, the proteoglycan syndecan, a transmembrane HS proteoglycan (HSPG) expressed on neurons, modulates signaling by the secreted guidance cue slit. Syndecan is also a ligand for the LAR receptor protein tyrosine phosphatase, regulating axonal pathfinding and synapse formation (Johnson et al. 2004; Steigemann et al. 2004; Fox and Zinn 2005).

Semaphorin proteins are phylogenetically conserved and include both secreted and transmembrane guidance cues (Tran et al. 2007). Semaphorins are also modulated by GAGs since HSPGs and CS proteoglycans (CSPGs) are required by the murine class 5 semaphorin Sema5A for axon guidance functions (Kantor et al. 2004). Moreover, the growth cone-collapsing activity of a vertebrate-secreted semaphorin, Sema3A, is enhanced by the addition of heparin (De Wit et al. 2005). However, in vivo evidence demonstrating that a specific proteoglycan can modulate semaphorin neuronal signaling is lacking.

Here, we show that the secreted HSPG perlecan is required for embryonic motor axon guidance in Drosophila. perlecan- and semaphorin-1a (Sema-1a)-null mutants exhibit similar motor axon guidance phenotypes, and we found that perlecan modulates Sema-1a repulsive signaling in vivo. Therefore, a secreted ECM protein modulates transmembrane guidance cue signaling during nervous system assembly and plays a key role in the establishment of neural connectivity during development.

\section{Results}

A Sema-1a gain-of-function (GOF) genetic screen on the $X$ chromosome identifies perlecan, a secreted HSPG, as a facilitator of Sema-1a-Plexin A (PlexA) signaling

In each segment of the Drosophila embryonic ventral nerve cord, commissural axons cross the CNS midline, forming one anterior and one posterior tract (Fig. 1A; Seeger et al. 1993). Sema-1a, a transmembrane semaphorin, can serve as a repellent required for CNS axon tract formation (Yu et al. 1998). Ectopic expression of Sema-1a in CNS midline glial cells in a Sema-1a-null mutant background (referred to here as "PUP" [Sema-1 $a^{\mathrm{P} 1}$, UAS-Sema-1a/+, P52-Gal4/+]), provides a sensitized genetic background in which most posterior commissural axons do not cross the midline: a robust Sema-1a GOF phenotype (Fig. 1B; Ayoob et al. 2004). In Drosophila, PlexA is a Sema-1a receptor (Winberg et al. 1998), and when a single PlexA-null allele was introduced into the PUP background, we observed significant suppression (Fig. 1C; Ayoob et al. 2004). Conversely, introduction of a genomic construct containing a bacterial artificial chromosome (BAC) that includes the entire PlexA locus (PlexA-BAC) into the PUP background leads to dramatic
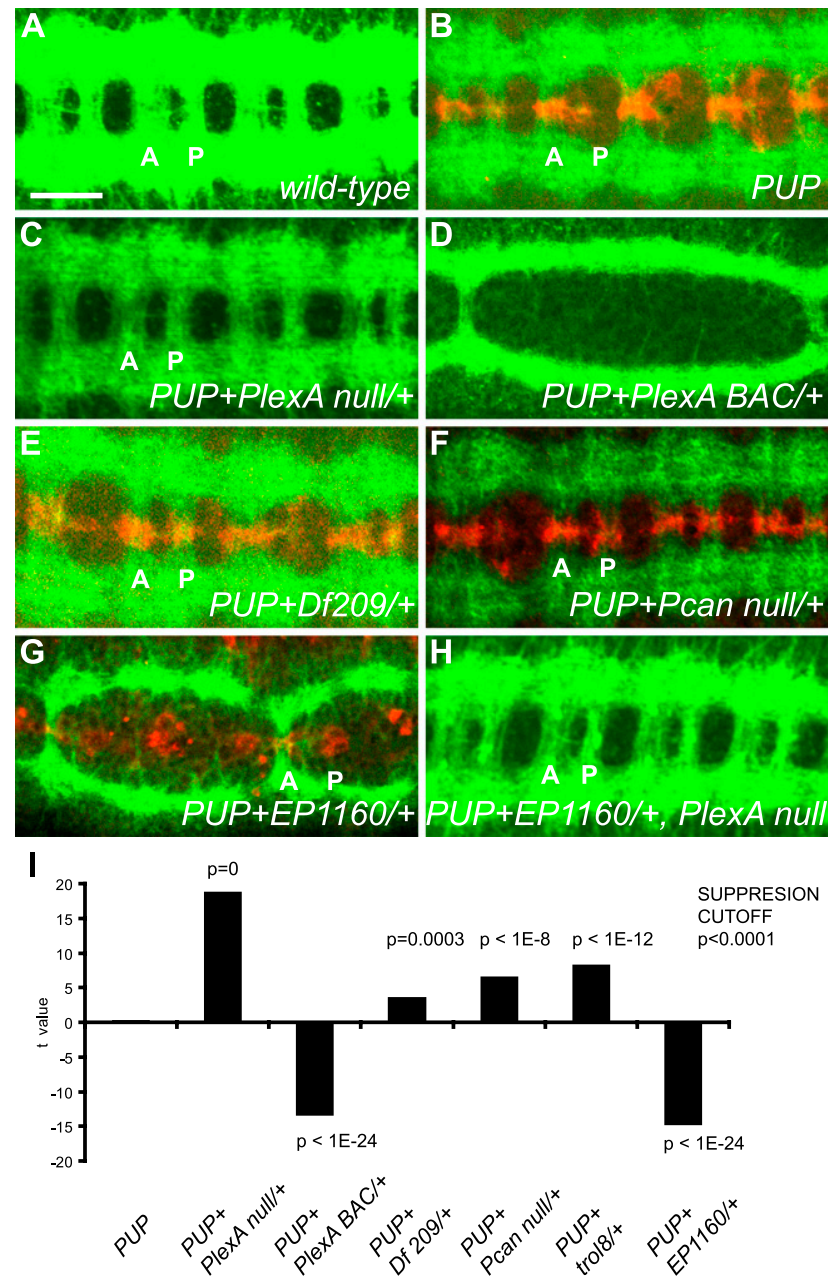

Figure 1. Sema-1a GOF screen on the X chromosome identifies perlecan as a facilitator of Sema-1a-PlexA signaling. $(A-H)$ Commissural axons stained with BP102 (green) and anti-Sema-1a antibody (red). Anterior is to the left. Bar, $20 \mu \mathrm{m}$. (A) In wild-type embryos, each segment contains anterior $(\mathrm{A})$ and posterior $(\mathrm{P})$ commissures. (B) Embryos misexpressing Sema-1a (red) in midline glia in a Sema-1 $a^{P 1}$ mutant background (PUP) lack posterior $(\mathrm{P})$ commissures, producing the Sema-1a GOF phenotype. $(C)$ Removing one copy of PlexA suppresses the Sema-1a GOF phenotype. $(D)$ Introducing the entire PlexA locus (PlexA-BAC) exhibits enhancement. (E) Df 209, a large deficiency that includes perlecan, shows suppression. $(F)$ perlecan-null mutants suppress the Sema$1 a$ GOF phenotype. $(G)$ Ectopic expression of perlecan results in enhancement. $(H)$ Ectopic expression of perlecan in a PlexA mutant results in no Sema-1a GOF phenotype. (I) Summary of Sema-1a GOF phenotypes measured in $t$-values (see the Materials and Methods for detailed scoring method). Positive and negative $t$-values indicate suppression and enhancement, respectively. $P$-values $<0.0001$ indicate significant suppression or enhancement (see the Materials and Methods for cutoff calculation methods).

enhancement (Fig. 1D). Here, we extended our use of the PUP background (Ayoob et al. 2004) to screen for novel components of the Sema-1a-PlexA signaling pathway on the X chromosome (see the Materials and Methods).

Heterozygosity for a PlexA-null mutant in the PUP background exhibited the strongest suppression (Supplemental 
Fig. 1A, red curve), whereas one copy of the PlexA-BAC showed the strongest enhancement (Supplemental Fig. 1A, green curve); control crosses resulted in neither suppression nor enhancement (Supplemental Fig. 1A, light-blue curve). We defined cutoffs that allowed us to determine which large deficiency lines significantly suppress or enhance the Sema-1a GOF phenotype (see the Supplemental Material). We crossed smaller deficiency lines, or candidate mutants, falling within the larger deficiencies back into the PUP background to identify genes responsible for suppression.

Among candidate genes (Supplemental Fig. 1A,B), we found that two different alleles of trol (trols and Pcan ${ }^{\text {null }}$ ) significantly suppress the Sema-1a GOF phenotype (Fig. 1E,F,I; Supplemental Fig. 1A [orange curves], B). We found that Df209, which includes trol, shows milder PUP suppression than any of the trol alleles (Fig. 1E,I), suggesting that other genes contained in Df 209 may negatively regulate Sema-1a signaling. Sema-1a ectopic expression levels on midline glia in the PUP background were not perturbed by loss of trol (Fig. 1B,E,F). Furthermore, mid-

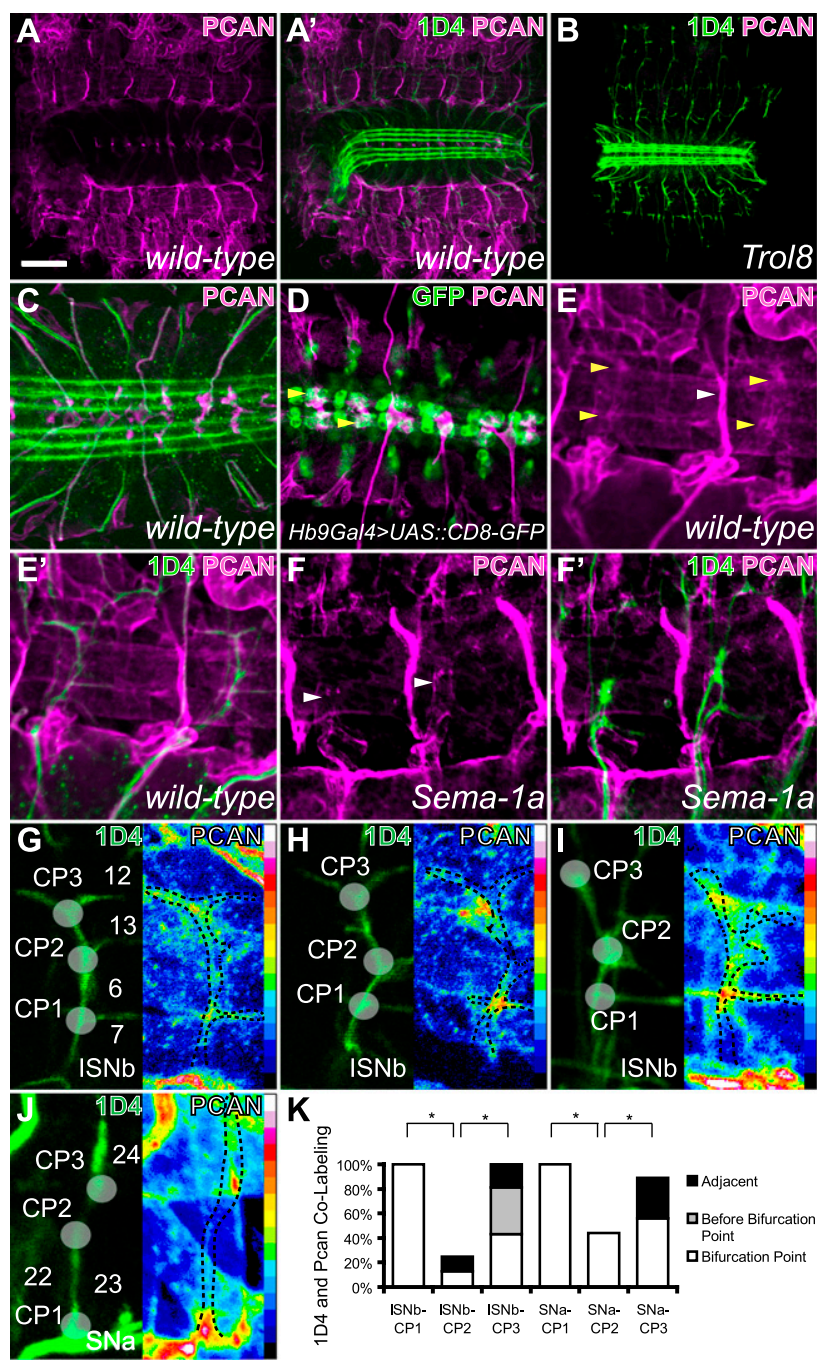

line glia do not exhibit aberrant morphology in trol mutants (Supplemental Fig. 1C,D).

The trol gene in Drosophila encodes a secreted HSPG called perlecan, an evolutionarily conserved ECM component (Friedrich et al. 2000; Voigt et al. 2002). Although Drosophila HSPGs modulate slit signaling (Johnson et al. 2004), none has been shown to modulate secreted or transmembrane semaphorin function in vivo. trol suppression of the PUP phenotype suggests that perlecan facilitates Sema-1a-PlexA signaling. Therefore, we crossed line EP1160, which harbors a $\mathrm{P}$ element that includes UAS sequences and is located just upstream of the "RG" perlecan isoform transcriptional start site (Fig. 4A), into the PUP background. We observed robust enhancement (Fig. 1G,I), confirming that perlecan facilitates Sema-1a repulsive signaling. This enhancement is completely absent in EP1160; PUP embryos that lack PlexA (Fig. 1H). Taken together, these data show that perlecan can facilitate Sema-1a-mediated axon repulsion in vivo.

Increased levels of perlecan are found in the vicinity
of motor axon trajectories and pathway choice points

Perlecan is expressed within the ventral nerve cord and in the embryonic body wall, and anti-perlecan staining is absent in perlecan-null mutants (Fig. 2A,B; Friedrich et al. 2000). Perlecan is highly expressed in the dorsal median cells, in close proximity to the medial and intermediate fasciclin- $\mathrm{II}^{+}\left(\mathrm{FasII}^{+}\right)$longitudinal connectives (Fig. 2C),

Figure 2. Perlecan is expressed in the periphery, with increased levels in the vicinity of motor axon trajectories and pathway choice points. $\left(A-F^{\prime}\right)$ Dorsal views of filleted stage 16 embryos. $\left(A-C, E-F^{\prime}\right)$ Embryos are stained with anti-perlecan (magenta) and anti-FasII mAb 1D4 (green). $\left(A, A^{\prime}\right)$ Perlecan expression in wild-type embryos. Bar, $60 \mu \mathrm{m}$. (B) Lack of anti-perlecan staining in trols mutants. Bar, $60 \mu \mathrm{m}$. $(C)$ In wild-type embryos, perlecan CNS expression is in close proximity to the medial and intermediate $\mathrm{FasII}^{+}$longitudinal connectives. Bar, $20 \mu \mathrm{m}$. $(D)$ In HB9-Gal4 animals ectopically expressing CD8-GFP, perlecan (magenta) is colocalized with GFP where the cell bodies of Islet ${ }^{+}$ $\mathrm{RP} 1 / 3 / 4 / 5$ motoneurons reside (yellow arrow head). Bar, $20 \mu \mathrm{m}$. $\left(E, E^{\prime}\right)$ Perlecan is highly expressed in muscle attachment sites (white arrowhead), and elevated expression of perlecan is detected along the ISNb (green) motor axonal trajectory (yellow arrowheads). Bar, $10 \mu \mathrm{m}$. $\left(F, F^{\prime}\right)$ In Sema-1 $1 a^{P 1}$ mutant embryos, much weaker perlecan expression is observed along the ISNb axonal trajectory (white arrowheads). Bar, $10 \mu \mathrm{m}$. $(G-I)$ In each panel, on the left is a single ISNb pathway stained with 1D4 antibody (green) and labeled to show the three choice point areas (CP1-3) where axons defasciculate (white translucent circles). At the right of each panel is shown the fluorescence intensity of perlecan expression, based on staining with anti-perlecan, in a heat map format. Bar, $5 \mu \mathrm{m}$. $(G)$ Elevated perlecan expression is detected at the bifurcation points $\mathrm{CP} 1$ and $\mathrm{CP} 3$ : adjacent to $\mathrm{CP} 3$ and at the bifurcation point in $\mathrm{CP} 1(H)$, and at the bifurcation point in CP1 and before bifurcation in CP3 $(I)$. (J) The SNa pathway stained with 1D4 (green) and anti-perlecan antibody (heat diagram depicts staining intensity). (K) Correlation of perlecan localization with choice point areas (CP1-3) along the ISNb and SNa pathways. $\left(^{\star}\right) P<0.01$. 
and also in CNS regions where commissures cross the midline (Supplemental Fig. 2A). We performed immunohistochemistry (IHC) for perlecan in HB9-Gal4, CD8-GFP embryos. In these embryos, CD8-GFP is expressed in subsets of motor neurons, including the Islet ${ }^{+} \mathrm{RP} 1 / 3 / 4 / 5$ motoneurons that innervate the ventral lateral muscle field via the intersegmental nerve $\mathrm{b}$ (ISNb) (Odden et al. 2002). Interestingly, perlecan partially colocalizes with these motoneuron cell bodies (Fig. 2D, yellow arrowheads; Supplemental Fig. 2B-B").

Motor nerves exiting the CNS in each hemisegment include the intersegmental nerve (ISN) and segmental nerve (SN) (Fig. 3G; Landgraf et al. 1997). ISNb motor axons defasciculate from the main ISN bundle and then navigate along ventral lateral muscles $6,7,12$, and 13 (Figs. 2G, 3G). There are three major ISNb pathway choice points, and they are numbered, medial to lateral, $\mathrm{CP} 1, \mathrm{CP} 2$, and CP3; they are located between muscles 6 and 7, 13 and 6, and 12 and 13, respectively (Fig. 2G). At CP1, RP3 neurons defasciculate between muscles 6 and 7 . At CP2, RP1 and RP4 axons defasciculate, and the remaining RP5 axons extend dorsally between muscles 13 and 12, extending processes anteriorly and posteriorly (Figs. 2G, 3G). Axons in the dorsal branch of the $\mathrm{SNa}$ pathway innervate muscles 22, 23, and 24 (Figs. 2J, 3G; Landgraf et al. 1997).
Although perlecan is expressed in several regions of the ventral-lateral muscle field, elevated levels of perlecan are observed along motor axon trajectories in close proximity to the ISNb pathway (Fig. 2E-E', yellow arrowheads). It is not clear whether perlecan is secreted from muscle, glia, or neurons. However, the pattern of elevated perlecan expression along the ISNb trajectory is less robust in Sema-1a mutants, where ISNb motor axons are severely stunted and often do not reach their final targets (Fig. 2F-F', white arrowheads).

To investigate perlecan distribution along the ISNb, we quantitatively assessed perlecan protein distribution using $\mathrm{mAb} 1 \mathrm{D} 4$ to illuminate motor neurons and antiperlecan. This allowed us to evaluate the correlation between perlecan localization and motor axon choice points. Elevated perlecan levels are detected at CP1 in almost all embryos (Fig. 2G-I,K). This is the branch point where motor axon defasciculation is dramatically compromised in the absence of Sema-1A-PlexA signaling (Winberg et al. 1998; Yu et al. 1998). At CP2, we observed a reduced correlation, but at CP3, perlecan is found in close proximity to this choice point in most embryos; however, the CP3 perlecan location does not always correlate precisely with the ISNb bifurcation point (Fig. 2G$\mathrm{I}, \mathrm{K})$. We also observed a significant correlation between perlecan expression and SNa choice points: strong corre-

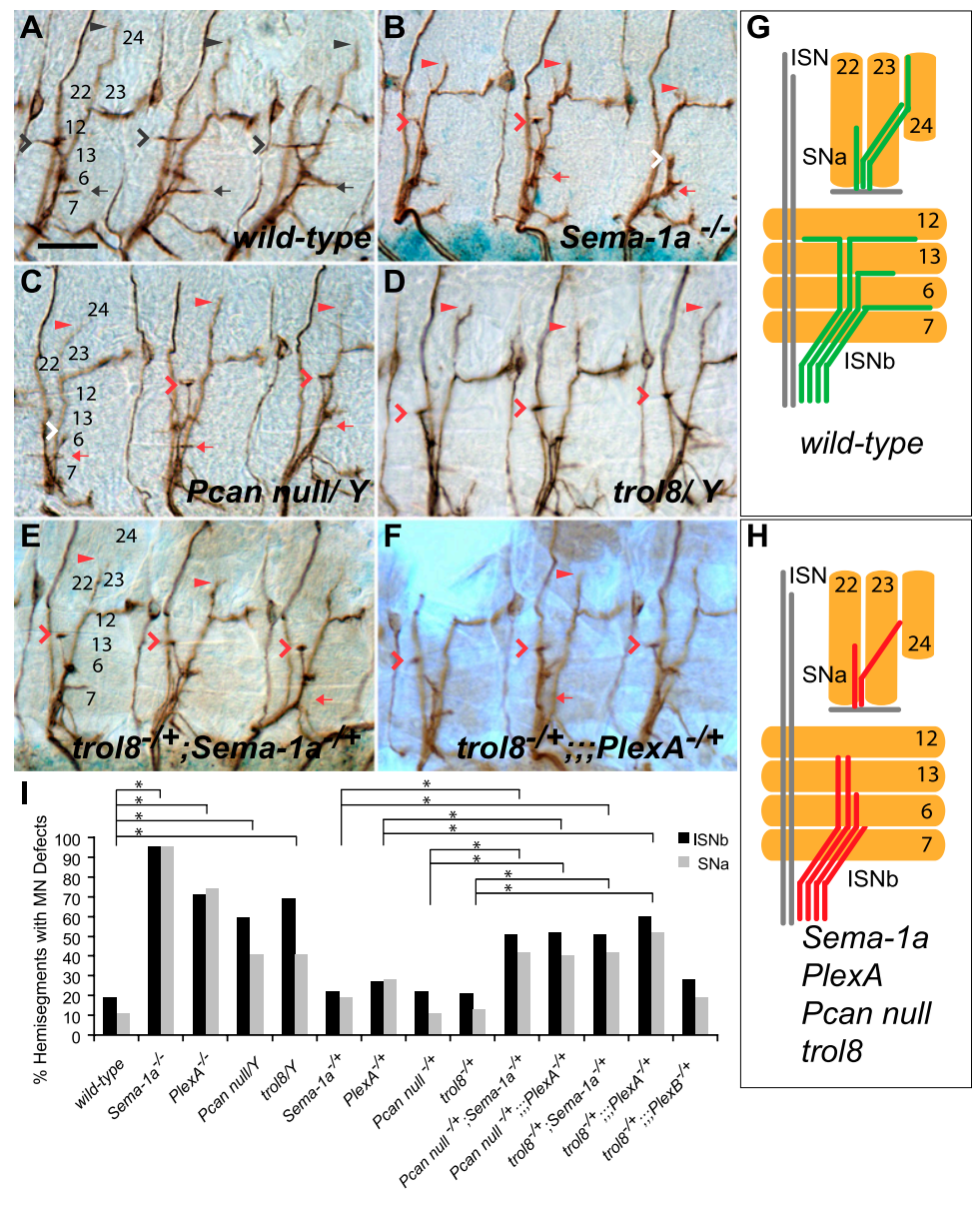

Figure 3. Perlecan is required for Sema-1a-mediated motor axon defasciculation. $(A-F)$ Three hemisegments stained with 1D4 antibody. Anterior is at the left, and lateral is at the top. Bar, $20 \mu \mathrm{m}$. (A) In wild-type embryos, ISNb axons navigate dorsally and defasciculate at muscles $6 / 7$ (black arrows) and at muscles 12/13, sending axons both anteriorly and posteriorly (black notches). SNa axons defasciculate between muscles 22/ 23, grow dorsally, and innervate muscle 24 (black arrowheads). (B) In Sema-1 $1 a^{P 1}$ mutants, axons comprising the ISNb pathway often fail to defasciculate at muscles 6/7 (red arrows) and 12/13 (red notches); in severe cases, axons stall between muscles 6/13 (white notch). Axons within the SNa pathway fail to defasciculate between muscles $22 / 23$ (red arrowheads). $(C, D)$ In perlecan mutants, ISNb axons fail to defasciculate (red notches) at muscles 12/13 and often lack innervation at muscles 6/7 (red arrows); some axons stall between muscles 6/13 (white notch). SNa axons also fail to reach their proper targets (red arrowheads). $(E, F)$ perlecan genetically interacts with Sema-1a and PlexA. Axons within the ISNb fail to defasciculate at muscles 6/7 (red arrows) and 12/13 (red notches), and SNa axons also fail to reach their targets (red arrowheads) in embryos transheterozygous for trol $^{-/+} ;$Sema- $1 a^{-/+}$ and trol $^{-/+} ; ;$Plex $A^{-/+} .(G, H)$ Schematic diagram of the ISNb and SNa pathways. (I) Quantitative summary of axon guidance defects in each genotype. $\left(^{\star}\right) P<0.01$. 
Cho et al.

lation at CP1 and CP3, and weaker correlation at CP2 (Fig. 2J,K). These data show that perlecan localization is concentrated at motor axon choice points, and so perlecan is well-positioned to facilitate Sema-1a-mediated motor axon defasciculation events.

\section{Perlecan is required for motor axon defasciculation} during embryonic development

Sema-1a is expressed along most, if not all, motor axon bundles, and in Sema- $1 a^{P 1}$ mutant embryos, many ISNb axon bundles stall at $\mathrm{CP} 2$, unable to send remaining ISNb motor axons dorsally, with some failing to defasciculate at CP3 (Fig. 3B,I; Yu et al. 1998). PlexA mutants exhibit similar motor axon guidance phenotypes (Fig. 3I; Winberg et al. 1998). To ask whether perlecan is required for motor axon guidance, we examined two different trol alleles (Voigt et al. 2002; Park et al. 2003), both of which significantly suppress the Sema-1a GOF PUP phenotype
(Fig. 1I). Indeed, both Pcan ${ }^{\text {null }}$ and trol8 mutants display motor axon guidance defects similar to those seen in Sema- $1 a^{P 1}$ and PlexA mutants, although with somewhat lower penetrance $\left(\right.$ Pcan $^{\text {null }}, 59.9 \%$ total ISNb defects; trol8, 69.1\%; Sema-1 $a^{P 1}, 95.5 \%$; and PlexA, 70.8\%) (Fig. $3 \mathrm{C}, \mathrm{D}, \mathrm{H}, \mathrm{I}$; Table 1). Most of the ISNb guidance defects observed in trol mutants include RP5 and RP3 defasciculation failures (data not shown). The perlecan phenotypes that we observed occur in those regions of the ISNb trajectory where we found that perlecan protein expression is enriched: at CP1 and CP3. We did not observe any differences in glial cell nucleus number or positioning (Supplemental Fig. 3C,D) or in glial ensheathment of ISNb motor axons (Supplemental Fig. 3A,B) in perlecan mutants. Moreover, overall muscle organization in perlecan mutants is indistinguishable from wild type (Supplemental Fig. 3E,F).

To ask whether perlecan functions in Sema-1a-PlexA repulsive signaling, we looked for genetic interactions in

Table 1. Summary of motor axon guidance phenotypes

\begin{tabular}{|c|c|c|c|}
\hline Genotype & $\begin{array}{c}\text { Abnormal ISNb } \\
\text { pathway }^{\text {a-d }}\end{array}$ & $\begin{array}{c}\text { Abnormal SNa } \\
\text { pathway }^{\mathrm{e}}\end{array}$ & $\begin{array}{c}\text { Hemisegments scored }^{f} \\
(x, y)\end{array}$ \\
\hline \multicolumn{4}{|l|}{ Controls } \\
\hline Wild type (w1118) & 18.9 & 10.6 & 133,144 \\
\hline Sema-1a $a^{P 1} /+$ & 22.2 & 18.7 & 108,107 \\
\hline $\operatorname{Plex} A^{d f(4) c 3} /+$ & 27.1 & 27.7 & 96,94 \\
\hline $\mathrm{PlexB}^{\mathrm{KG00878} /+}$ & 12.6 & 24.4 & 101,98 \\
\hline $\operatorname{trol}^{8} /+$ & 20.7 & 13.1 & 102,105 \\
\hline $\operatorname{Pcan}^{\text {null }} /+$ & 22.1 & 10.8 & 77,74 \\
\hline$S d c /+$ & 8.0 & 10.0 & 50,50 \\
\hline \multicolumn{4}{|l|}{ Loss of function } \\
\hline Sema-1 $a^{P 1} /$ Sema- $1 a^{P 1}$ & 95.5 & 95.2 & 110,104 \\
\hline $\operatorname{Plex} A^{d f(4) c 3} / P \operatorname{lex} A^{d f(4) c 3}$ & 70.8 & 74.2 & 96,97 \\
\hline $\operatorname{trol}^{8} / Y$ & 69.1 & 41.1 & 97,95 \\
\hline $\operatorname{Pcan}^{\text {null }} / Y$ & 59.9 & 38.7 & 279,266 \\
\hline PlexB $B^{K G 00878} /$ PlexB $B^{K G 00878}$ & 53.3 & 32.5 & 199,194 \\
\hline \multicolumn{4}{|l|}{ Rescue } \\
\hline Pcan ${ }^{\text {null }} / Y_{;} ; U A S-P c a n-R G /+$, elav-gal4/+ & 27.5 & 20.2 & 102,99 \\
\hline Pcan $^{\text {null }} / Y_{;}$UAS-Pcan-RG/+, repo-gal4/+ & 43.9 & 25.8 & 98,97 \\
\hline Pcan ${ }^{\text {null }} / Y_{;} ; U A S-P c a n-R G /+$, twi-gal4/+ & 57.6 & 46.3 & 85,80 \\
\hline Pcan $^{\text {null }} / Y ;$ UAS-Pcan-RG/+, repo-gal4/+,elav-gal4/+ & 25.7 & 21.2 & 101,99 \\
\hline \multicolumn{4}{|l|}{ Genetic interactions } \\
\hline $\operatorname{trol}^{8} /+; \operatorname{Sema}-1 a^{P 1} /+$ & 50.5 & 44.8 & 97,96 \\
\hline $\operatorname{trol}^{8} /+; ; P \operatorname{Plex} A^{d f(4) c 3 /+}$ & 60.2 & 52.4 & 108,103 \\
\hline $\operatorname{trol}^{8} /+, ; ; P l e x B^{K G 00878} /+$ & 28.0 & 19.0 & 100,100 \\
\hline Pcan $^{\text {null }} /+;$ Sema $-1 a^{P 1} /+$ & 51.0 & 41.8 & 153,146 \\
\hline $\operatorname{Pcan}^{\text {null }} /+; ;$ PlexA $A^{d f(4) c 3} /+$ & 51.5 & 40 & 97,95 \\
\hline Sdc/+, Sema-1a $a^{P 1} /+$ & 25.6 & 25.9 & 86,81 \\
\hline \multicolumn{4}{|l|}{ Gain of function } \\
\hline UAS-dally/+, elav-gal4/+ & 53.8 & 25.5 & 104,102 \\
\hline UAS-Sema-1a/+;5053-ga14/+ & $21.3[95.7,0,0]$ & $\mathrm{N} / \mathrm{A}$ & 108 \\
\hline UAS-Pcan/Y;;5053-gal4/+ & $18.1[94.1,0,0]$ & $\mathrm{N} / \mathrm{A}$ & 94 \\
\hline UAS-Pcan/Y;UAS-Sema-1a/+;5053-gal4/+ & $61.9[68.6,12.8,14.0]$ & $\mathrm{N} / \mathrm{A}$ & 139 \\
\hline
\end{tabular}

${ }^{\mathrm{a}} \mathrm{ISNb}$ defects (percentage hemisegments): The axons fail to separate from the ISN, stall between 6/13, or lack defasciculation at 6/7 or $12 / 13$.

${ }^{b}$ Percentage of ISNb axons lacking defasciculation at 12/13.

${ }^{\mathrm{c}}$ Percentage of ISNb axons making bulb figure.

${ }^{\mathrm{d}}$ Percentage of ISNb axons bifurcating aberrantly.

${ }^{\text {e}} \mathrm{SNa}$ defects (percentage hemisegments): The dorsal branch fails to separate from the lateral, makes detour, or lacks defasciculation between $22 / 23$ or $23 / 24$.

${ }^{f}(x, y)$ Number of hemisegments scored for ISNb $(x)$ and SNa $(y)$. 
multiple transheterozygous genotypes. Compared with Sema-1 $a^{P 1} /+$, PlexA/+, Pcan ${ }^{\text {null }} /+$, or trol8/+ single heterozygote embryos, all of which exhibit few to no motor axon guidance defects, $P$ can ${ }^{\text {null }} /+$;Sema-1a $1 a^{P 1} /+$, trol8/+; Sema-1a $a^{P 1} /+$, Pcan ${ }^{\text {null }} /+, ; ;$ PlexA $/+$ and trol8/+,;;PlexA/+ transheterozygous embryos all show significant ISNb and SNa motor axon guidance defects (Fig. 3E,F,I; Table 1). A PlexB-null mutation, which removes the secreted Sema-2a and Sema-2b receptor (Ayoob et al. 2006; Wu et al. 2011), did not exhibit genetic interactions with trol8 (Fig. 3I). Taken together, these perlecan loss-of-function (LOF) and genetic interaction data suggest that perlecan modulates transmembrane Sema-1a-mediated axon-axon repulsion.

\section{Cell type-specific rescue of perlecan mutants}

We next cloned the full-length perlecan cDNA (see the Materials and Methods) and examined in which cell types perlecan is required for motor neuron guidance. We cloned the two alternatively spliced transcripts, called " $R G^{\prime \prime}$ and " $R D$," that are located $\sim 5 \mathrm{~kb}$ and $\sim 13 \mathrm{~kb}$, respectively, downstream from the EP1160 insertion site (Fig. 4A, RG [red] and RD [green]). Expression of both the $R G$ and $R D$ cDNAs strongly enhances the Sema-1a GOF phenotype (Fig. 4B-E). Therefore, both perlecan cDNAs are sufficient for facilitating Sema-1a signaling despite different signal sequences and the $\sim 200$-aminoacid sequence difference at the $\mathrm{N}$ termini of the perlecan proteins encoded by $R G$ and $R D$.

We next expressed the $R G$ perlecan isoform in all neurons and detected robust pan-neuronal perlecan protein distribution when expression was driven by elavGal4 in a perlecan mutant (Fig. 4F). Neuronal perlecan expression significantly rescued the motor axon guidance phenotypes observed in Pcan $^{\text {null }}$ mutants $159 \%$ ISNb defects in $P$ can $^{\text {null }} / Y$ embryos rescued to $28 \%$ in Pcan $^{\text {null }} / Y$; elav-gal; UAS-Pcan-RG/+ embryos) (Fig. 4G,L). In contrast, robust expression of perlecan in all glial cells, including in glial processes that ensheath both ISNb and $\mathrm{SNa}$ pathways (Fig. 4H), only very modestly rescues Pcan null mutant motor axon defects (59\% ISNb defects in $P$ can ${ }^{\text {null }} / Y$ embryos to $44 \%$ in Pcan ${ }^{\text {null }} / Y$;repo-gal4;UAS-Pcan-RG/+ embryos) (Fig. 4H,I). Furthermore, we observed no rescue of Pcan $^{\text {null }}$ phenotypes when perlecan was ectopically expressed in mesodermal cells $158 \%$ defects observed in Pcan $^{\text {null }} / Y$; twi-gal4, UAS-Pcan-RG/+ embryos). This is somewhat surprising, since perlecan, a secreted HSPG expressed under control of the twi-Gal4 driver, is robustly expressed in the vicinity of developing motor axon trajectories (Fig. 4J). These results suggest that spatial regulation of perlecan expression is important for motor axon guidance at trajectory choice points. Furthermore, expression in motor axons and, to a much lesser extent, in glia apparently better mimics the wild-type perlecan distribution as compared with mesodermal expression.

\section{Perlecan enhancement of CNS and motor axon Sema-1a-PlexA signaling}

We next used a different PlexA GOF paradigm whereby neuronal expression of PlexA, driven by elav-Gal4, causes
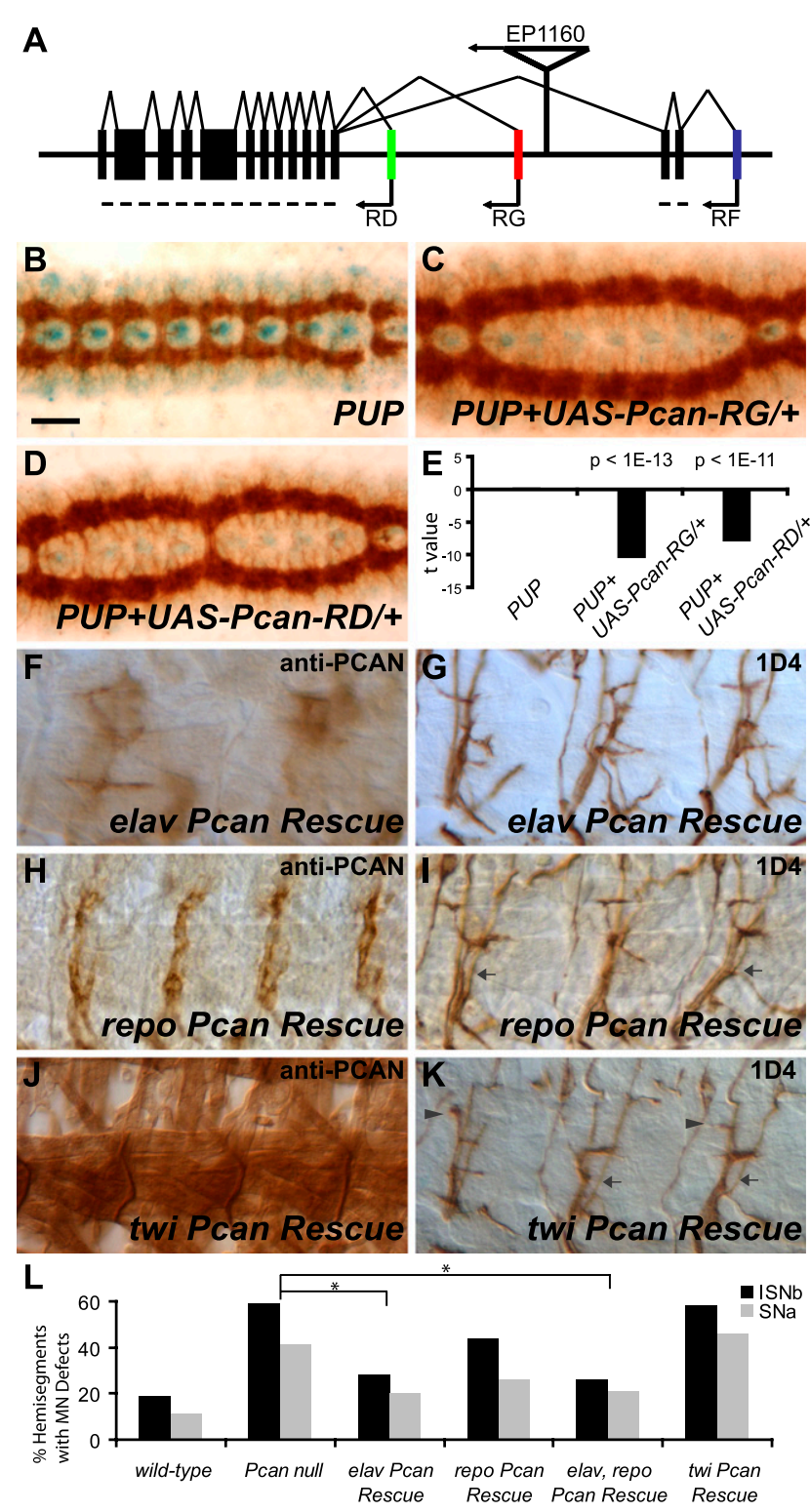

Figure 4. Cell type-specific rescue of perlecan mutants with functional perlecan cDNA. (A) Schematic representation of the perlecan genomic organization. The $5^{\prime}$ exons of the $R D, R G$, and $R F$ isoforms are shown in green, red, and blue, respectively. (B$D)$ Anterior is to the left, and posterior on the right. Bar, $20 \mu \mathrm{m}$. (B) PUP background embryos. $(C, D)$ Both $R G$ and $R D$ perlecan isoforms significantly enhance the PUP Sema-1a GOF phenotype. $(E)$ Summary of $B-D$. Embryos stained with anti-perlecan antibody $(F, H, J)$ and with 1D4 antibody $(G, I, K)$ are shown. Bars, $15 \mu \mathrm{m}$. (F) Perlecan is detected along the axonal trajectories and at choice points of the ISNb pathway in Pcan ${ }^{\text {null }}$ mutants rescued with a pan-neuronal driver. Bar, $8 \mu \mathrm{m}$. $(G) P_{\text {Pcan }}^{\text {null }}$ mutants rescued with a pan-neuronal driver. $(H)$ Pan-glial rescue in $P_{c a n}{ }^{\text {null }}$ shows peripheral glial expression of perlecan. (I) ISNb axons lacking defasciculation at muscles 6/7 (black arrows) in pan-glial rescue animals. ( $J$ ) Perlecan muscle expression in mesodermal rescue of Pcan ${ }^{\text {null }}$ mutants. (K) ISNb pathways lack defasciculation at muscles 6/7 and 12/13 (black arrows and arrowheads, respectively) in mesodermal perlecan rescue animals. $(L)$ Summary of cell type-specific rescue experiments. $\left({ }^{\star}\right) P<0.01$. 
Cho et al.

CNS medial longitudinal tracts that normally never cross the CNS midline to aberrantly cross at a low, but significant, frequency (Fig. 5A,B; Ayoob et al. 2004). Simultaneously overexpressing PlexA and perlecan in all neurons significantly enhances this aberrant CNS midline crossing phenotype (Fig. 5C,D), in line with our previous observations showing that neuronal perlecan is capable of enhancing PlexA signaling following Sema-1a GOF in CNS midline glia (Fig. 1C).

We further addressed whether perlecan facilitates Sema-1a-PlexA forward signaling in motor axons by providing an ectopic source of Sema-1a in trans using the 5053A-Gal4 driver (Ritzenthaler et al. 2000) to express Sema-1a, perlecan, or both proteins only in the ventral longitudinal muscle 12 (Fig. 5E). Overexpressing Sema-1a or perlecan in muscle 12 using one copy of either trans-
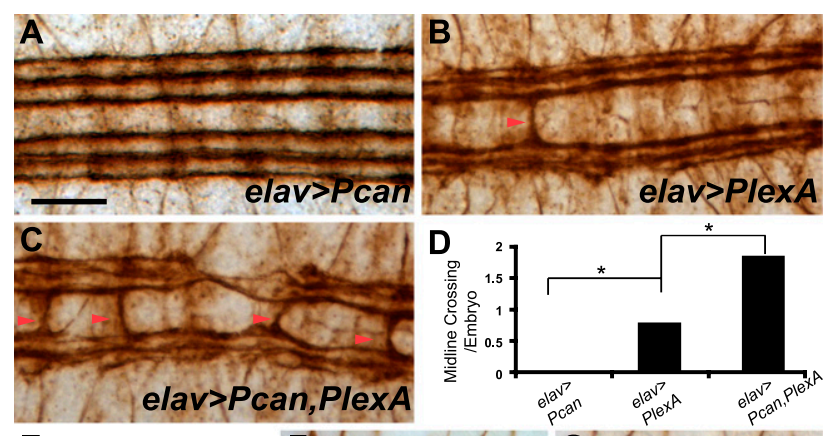

E
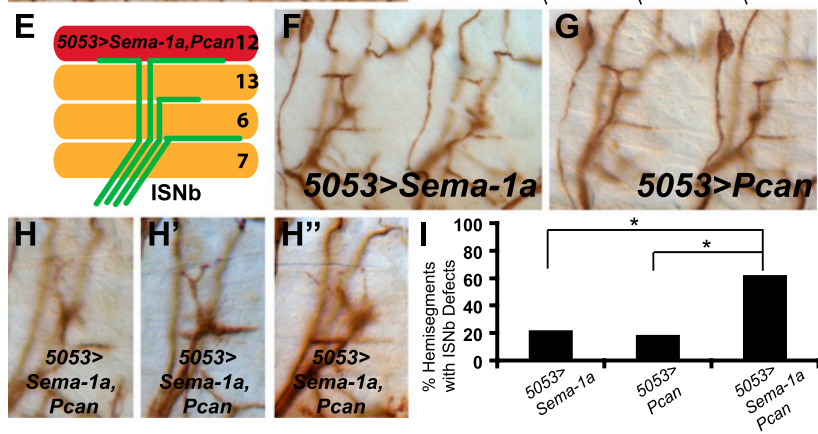

Figure 5. Perlecan facilitation of Sema-1a-PlexA signaling in the CNS and in motor axons. $(A-C)$ Embryos stained with 1D4 antibody. Bar, $20 \mu \mathrm{m}$. (A) Longitudinal axonal tracts never cross the midline in embryos ectopically expressing perlecan in all neurons. (B) Pan-neuronal expression of PlexA results in rare, but significant, abnormal CNS midline crossing (red arrowhead). (C) Overexpressing perlecan with PlexA in the nervous system significantly increases the frequency of abnormal midline crossing (red arrowheads). (D) Average number of midline crossings per embryo. $\left.{ }^{\star}\right) P<0.01$. (E) Schematic diagram of the ISNb axon pathway and RP5 axon bundles encountering muscle 12. $\left(F-H^{\prime \prime}\right)$ Embryos stained with 1D4 antibody. Anterior is to the left, and lateral is to the top. Bar, $20 \mu \mathrm{m}$. $(F, G)$ Embryos ectopically expressing either Sema-1a or perlecan from muscle 12 in trans show few ISNb RP5 motor axon innervation defects. $\left(H-H^{\prime \prime}\right)$ Simultaneous overexpression of Sema-1a and perlecan from muscle 12 results in a significant increase in RP5 defasciculation defects at the most dorsal choice point (CP3) and also targeting defects, resulting in premature bifurcations. (I) Perlecan facilitates ectopic Sema-1a expression from muscle 12. $\left(^{\star}\right)$ $P<0.01$. gene alone did not significantly affect RP5 motor axon targeting to muscle 12 or defasciculation at the final choice point between muscles 12 and 13 (Fig. 5F,G). However, simultaneous overexpression of Sema-1a and perlecan from muscle 12 resulted in a significant increase in RP5 defasciculation defects (Fig. 5H) and targeting defects (Fig. $\left.5 \mathrm{H}^{\prime}, \mathrm{H}^{\prime \prime}\right)$, leading to premature motor axon bifurcation. These phenotypes most likely result from enhanced Sema-1a-mediated repulsion in trans augmented by perlecan coexpressed in muscle 12. Taken together, these GOF experiments show that perlecan can facilitate PlexA signaling in CNS interneurons and Sema-1a signaling in trans to motor axons.

\section{Specificity of HSPG-mediated modulation of Sema-1a signaling}

Several genes in Drosophila encode HSPGs, including the transmembrane HSPG syndecan, the GPI-linked HSPGs dally and dally-like, and the secreted HSPG perlecan (Van Vactor et al. 2006). In invertebrates, syndecan has been studied extensively in both Drosophila and Caenorhabditis elegans neural development and plays key roles in repulsive axon guidance mediated by slit-Robo signaling and in synaptogenesis at the neuromuscular junction (Johnson et al. 2004; Steigemann et al. 2004; Fox and Zinn 2005; Rawson et al. 2005). In Drosophila, dally-like has been implicated in visual system and CNS axon guidance (Johnson et al. 2004; Rawson et al. 2005). Both syndecan and dally-like are expressed on Drosophila embryonic CNS axons and are found in both the anterior and posterior commissures of each segment (Johnson et al. 2004). However, the expression of dally has not been presented.

We found that neither syndecan nor dally-like mutants suppressed or enhanced the PUP Sema-1a GOF phenotype (Fig. 6A). Furthermore, embryos transheterozygous for Sema-1a and syndecan mutant alleles do not show significant genetic interactions affecting motor axon guidance (Fig. 6B). In contrast, dally mutants significantly enhanced the PUP Sema-1a GOF phenotype, suggesting that dally may modulate Sema-1a signaling but, unlike perlecan, in a negative fashion. We ectopically expressed dally in all embryonic neurons in a wild-type background and observed an increased level of motor axon defasciculation defects in the ISNb pathway, reminiscent of mild Sema-1a LOF phenotypes (Fig. 6B). This suggests that dally and perlecan may act in opposition with respect to modulation of Sema-1a signaling.

We also considered whether perlecan modulates other axon guidance signaling pathways, particularly since perlecan CNS expression partially overlaps with the midline guidance cues slit and netrins A and B and also their receptors (Kolodziej et al. 1996; Mitchell et al. 1996; Johnson et al. 2004). perlecan mutants do not phenocopy the embryonic CNS midline crossing and recrossing of FasII $^{+}$longitudinal connectives characteristic of Robo-null mutants (Fig. 2B). In addition, we found that perlecan mutants do not exhibit any of the CNS or peripheral nervous system (PNS) guidance errors observed in frazzled mutants (Kolodziej et al. 1996). These data suggest that perlecan 
A

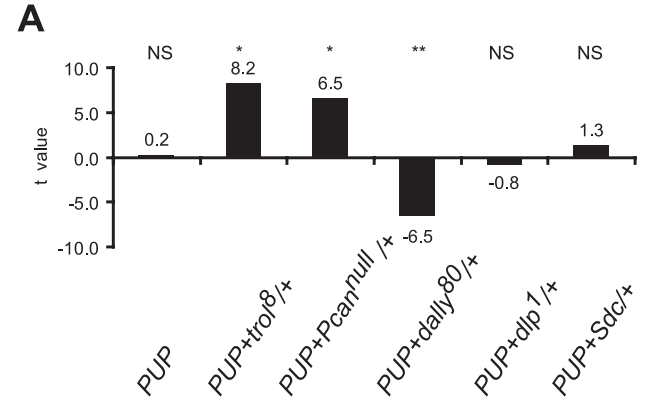

B

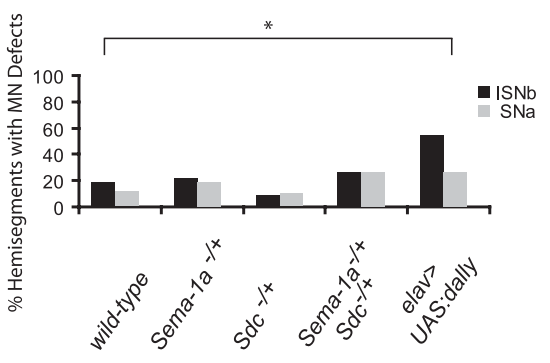

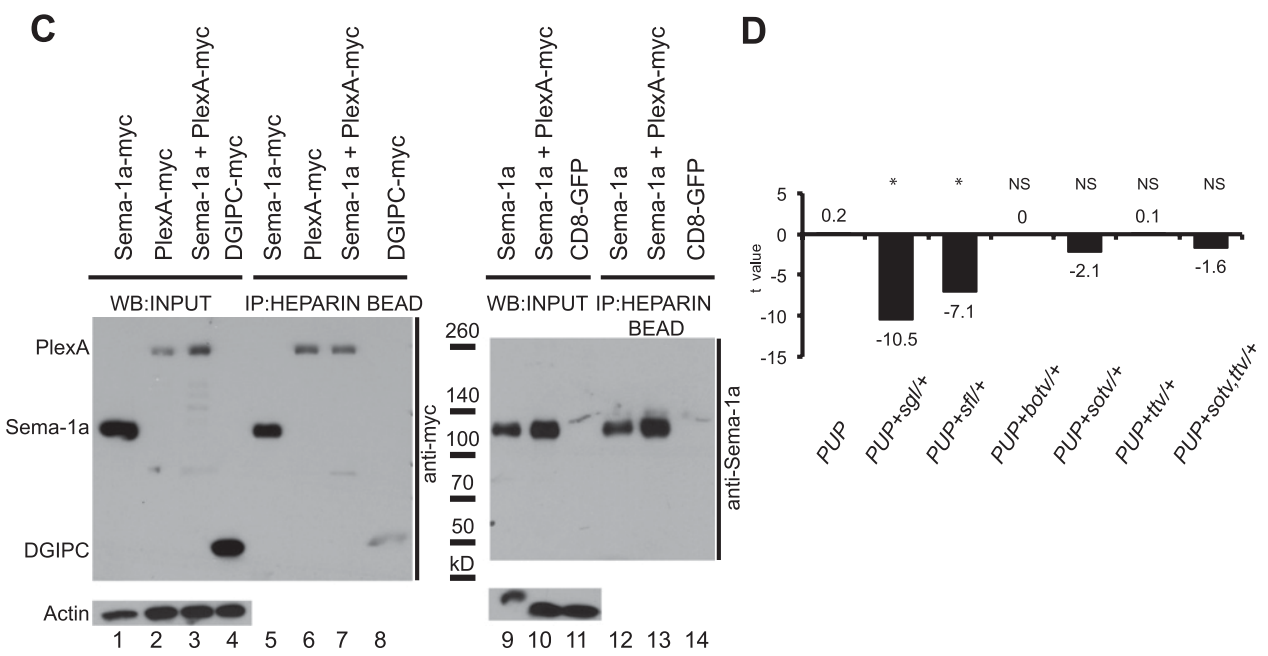

Figure 6. Specificity of HSPG-mediated Sema-1a signaling modulation and HS involvement in Sema-1a signaling. (A) Summary of $t$-values for PUP Sema-1a GOF assays with various HSPG mutants. Dlp ${ }^{1}$ (dally-like) or Sdc (syndecan) mutants do not suppress or enhance the Sema-1a GOF phenotype. However, dally ${ }^{80}\left(\right.$ dally) mutants enhance the Sema-1a GOF phenotype. Suppressing $\left(^{\star}\right)$ and enhancing $\left(^{* \star}\right)$ fly lines with $P<0.0001$; (NS) not significant, $P>0.0001$. (B) Summary of hemisegments with motor neuron pathfinding defects in both ISNb and SNa pathways for different genotypes. syndecan and Sema-1 $a^{P 1}$ mutants do not genetically interact. Panneuronal expression of dally results in increased levels of ISNb pathway innervation defects compared with wild type. $\left({ }^{\star}\right) P<0.01 .(C)$ PlexA and Sema-1a, but not dGIPC, bind to heparin beads. S2R ${ }^{+}$cells were transfected with the cDNAs indicated above each lane. Lanes 1-4 and 9-11 show Western blots from cell lysates, and lanes 5-8 and 12-14 display initial lysates that were subsequently incubated with heparin beads and then subjected to pull-down using these beads. Lanes 1-8 are blotted with anti-myc antibody to detect PlexA, Sema-1a, and dGIPC, and lanes 9-14 are blotted with anti-Sema-1a to detect Sema-1a. (WB) Western blot; (IP) immunoprecipitation. This experiment was performed twice. (D) Summary of $t$-values for Sema-1a GOF assays with HS biosynthetic and modifying enzymes. sgl and $s f 1$ mutants robustly enhance the Sema-1a GOF phenotype. $\left(^{*}\right) P<0.0001 ;$ (NS) $P>0.0001$.

does not play a major role in modulating endogenous embryonic netrin- or slit-mediated axon guidance, exhibiting specificity with respect to its role in regulating neuronal guidance cues.

\section{Perlecan HS chain involvement in Sema-1a signaling facilitation}

To investigate HS chain contribution to perlecan-mediated Sema-1a signaling, we first tested physical interactions between HS and Sema-1a or PlexA. Both Sema-1a and PlexA, but not the cytosolic control protein dGIPC, bound robustly to heparin-agarose beads (Fig. 6C, Sema-1a [cf. lanes 1 and 5, and lanes 9 and 12], PlexA [cf. lanes 2 and 6], and dGIPC [cf. lanes 4 and 8]). Moreover, cells coexpressing Sema-1a and PlexA showed similar levels of each protein binding to heparin beads, suggesting that HS does not negatively affect the association between this ligand and its receptor or vice versa (Fig. 6D, cf. lanes 3 and 7 , and lanes 10 and 13). Overall, these interactions suggest that HS chains in perlecan or dally may bind to Sema-la or PlexA directly.

Next, we assessed mutants that affect HS chain biosynthesis in the PUP background. Proteins with homology with UDP-glucose dehydrogenase, N-deacetylase/ $\mathrm{N}$-sulfotransferase, and EXT are encoded in Drosophila by sugarless (sgl), sulfateless (sfl), and EXT genes, respectively (Botv is required for HS chain initiation, and Ttv and Sotv are required for HS chain elongation) (Van Vactor et al. 2006). Sgl is required for the formation of glucuronic acid, which in turn is required for biosynthesis of HS, CS, and dermatan sulfate (Lin and Perrimon 1999). When HS chains are elongated, the enzyme $\mathrm{N}$-deacetylase/ $\mathrm{N}$-sulfotransferase, which in Drosophila is encoded by sfl, removes acetyl groups from GlcNAc residues and substitutes them with sulfate groups (Kamimura et al. 2011). 
Mutations in any one of the EXT genes affect HSPG biosynthesis in vivo, demonstrating that they are not functionally redundant (Takei et al. 2004). We introduced all five single mutants and also the ttv; sotv doublemutant combination into the PUP background. Interestingly, sfl and sgl mutations exhibited strong dominant enhancement of the Sema-1a GOF phenotype; however, none of the EXT mutants exhibited suppression or enhancement (Fig. 6D). The strong enhancement shown by $s f l$ and $s g l$ mutants suggests that HSs, presumably on perlecan or dally, act to inhibit Sema-1a signaling. In addition, sgl mutants exhibit stronger enhancement than $s f 1$ mutants $(s g l t$-value $=-10.5 ; s f 1$-value $=-7.1)$, raising the possibility that both HSs and CSs are involved in negatively regulating Sema-1a signaling. However, relatively stronger maternal effects could explain the lack of suppression or enhancement in EXT mutants. Given our biochemical and genetic data, HSs on perlecan or dally could bind to Sema-1a and PlexA directly and negatively regulate Sema-1a signaling in vivo.

\section{Perlecan enhances Sema-1a-mediated focal adhesion} kinase (FAK) dephosphorylation

During axon pathfinding, integrins function by selectively stabilizing contacts with permissive ECM substrates, including laminin and fibronectin (Robles and Gomez 2006). Since guidance cue-directed axon pathfinding likely includes regulation of integrin-based adhesion (Myers et al. 2011), cross-talk between semaphorin and integrin signaling has the potential to result in inhibition of integrin-mediated adhesion to the ECM, and vice versa (Serini et al. 2003; Oinuma et al. 2006; Tran et al. 2007).

Integrin receptors bind ECM components and often signal cytoskeletal changes through activation of FAK (Ivankovic-Dikic et al. 2000), and increased levels of FAK phosphorylation are often observed at sites of integrin activation and clustering (Kornberg et al. 1992). In Drosophila, phosphorylated FAK is absent from muscle attachment sites in integrin mutants, and FAK genetically interacts with integrin mutations (Grabbe et al. 2004). Moreover, changes in FAK phosphorylation levels have been observed upon semaphorin treatment (Pasterkamp et al. 2003; Oinuma et al. 2006; Schlomann et al. 2009). Therefore, we asked whether FAK phosphorylation levels might reflect cross-talk between Sema-1a and integrin signaling. We observed a dramatic decrease in phosphoFAK levels when the soluble extracellular domain of Sema-1a, at a concentration of $\sim 35 \mathrm{nM}$, was used to treat Drosophila S2R ${ }^{+}$cells in vitro for $30 \mathrm{~min}$ (Fig. 7A,B; lanes $1,5)$. However, this decrease was significantly attenuated when the cells were pretreated with PlexA dsRNA, strongly suggesting that this effect on FAK phosphorylation is specific to the Sema-1a-PlexA signaling pathway (Fig. 7A,B, lanes 5-7). PlexA is expressed in S2 $\mathrm{R}^{+}$cells, and we observed that the effect of PlexA dsRNA on PlexA protein levels is robust (Fig. 7A,B, lanes 2,3,6,7). Consistent with our in vivo genetic analyses, overexpressing perlecan in $\mathrm{S}_{2} \mathrm{R}^{+}$cells enhanced Sema-1a-mediated dephosphorylation of FAK by $\sim 40 \%(P=0.00095)$ (Fig. 7A,B, lanes 5,8$)$. Interestingly, overexpression of perlecan alone dramatically reduced phospho-FAK levels (Fig. 7A,B, lanes 1 ,4). However, $\mathrm{S} 2 \mathrm{R}^{+}$cells overexpressing perlecan and PlexA dsRNA showed no reduction of phospho-FAK levels, suggesting that perlecan-mediated reduction of phospho-FAK levels is dependent on PlexA signaling (Fig. 7C).

Since phospho-FAK levels are reduced upon Sema-1a treatment in vitro, we investigated whether Sema-1 $a$ and $F A K$ genetically interact in vivo. We did not observe any axon pathfinding defects in Fak56 $6^{C G 1}$ mutants (data not shown). However, robust genetic interactions were observed between $F A K$ and integrin in the developing wing disc when FAK was overexpressed in integrin heterozygotes (Grabbe et al. 2004). Therefore, we compared Sema$1 a^{P 1} /+$ heterozygous embryos to either wild-type or Sema-1 $1 a^{P 1} /+$ heterozygous embryos in which FAK was overexpressed in the nervous system. We observed that neuronal overexpression of $F A K$ alone results in the formation of severe gaps at high penetrance in the lateralmost $\mathrm{FasII}^{+} \mathrm{CNS}$ longitudinal fascicles and also aberrant CNS midline crossing of axons from the medial-most $\mathrm{FasII}^{+}$longitudinal connectives; these phenotypes are reminiscent of CNS defects observed following integrin LOF (Fig. 7F; Supplemental Fig. 4A, $\mathrm{A}^{\prime}, \mathrm{C}$; Hoang and Chiba 1998). Given that FAK is an integrin effector and $F A K$ GOF leads to integrin LOF phenotypes, FAK GOF may function in a dominant-negative fashion (Hoang and Chiba 1998). Interestingly, removing one copy of Sema-1a in this FAK GOF background significantly suppresses these CNS longitudinal tract defects, consistent with our in vitro observation that Sema-1a treatment down-regulates FAK phosphorylation and so appears to function antagonistically with respect to FAK activation. These results demonstrate a robust genetic interaction between Sema-1a and FAK GOF in vivo (Fig. 7F; Supplemental Fig. $\left.4 \mathrm{~B}, \mathrm{~B}^{\prime}, \mathrm{C}\right)$. One interpretation of these results is that endogenous Sema-1a signaling lowers phospho-FAK levels, but when FAK is overexpressed, non-phospho-FAK levels increase relative to phospho-FAK, thus leading to inhibition of integrin signaling. However, when one copy of Sema-1a is removed, relative levels of phospho-FAK increase, thereby restoring integrin function. These results suggest that the ability of Sema-1a to regulate phosphoFAK protein levels is important for axon guidance.

Sema-1a-PlexA modulation of FAK phosphorylation in vitro and also genetic interactions between Sema-1a and $F A K$ in vivo suggest that there exists cross-talk between Sema-1a and integrin signaling in vivo. Therefore, we asked whether mutations in genes encoding Drosophila integrin receptor components-if, mew, and mys ( $\alpha \mathrm{PS} 2$, $\alpha$ PS1, and $\beta$ PS subunits, respectively) (Bokel and Brown 2002)-modify the PUP Sema-1a GOF phenotype. Interestingly, we found that a mutation in if significantly enhanced the PUP Sema-1a GOF phenotype, and a mutation in mys exhibited a more modest, but significant, level of enhancement; however, mew mutants did not exhibit any enhancement or suppression (Fig. 7E). The reason why if but not mew shows PUP enhancement may be due to the specificity of the $\alpha$ PS2 subunit interaction 
A

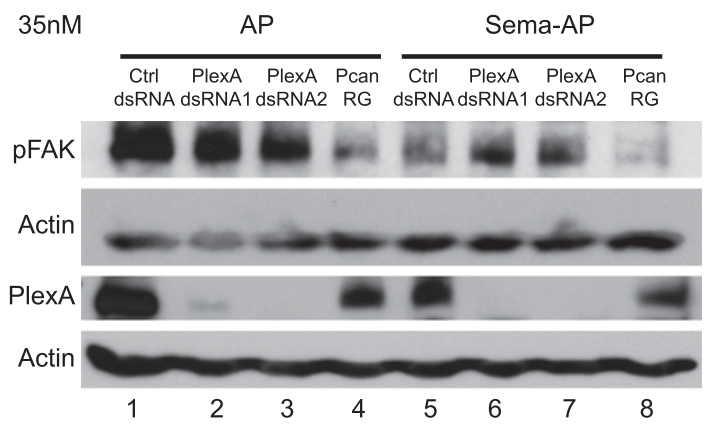

C

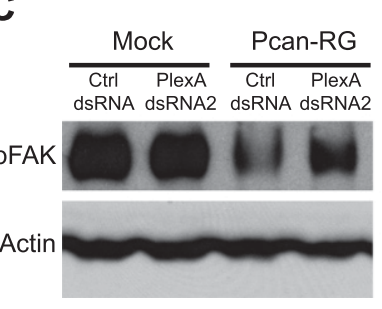

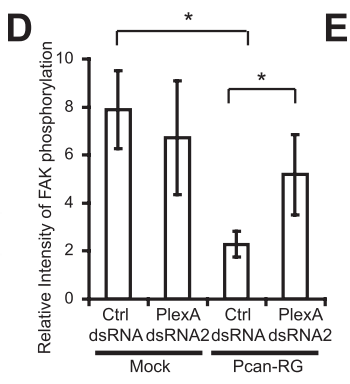

B

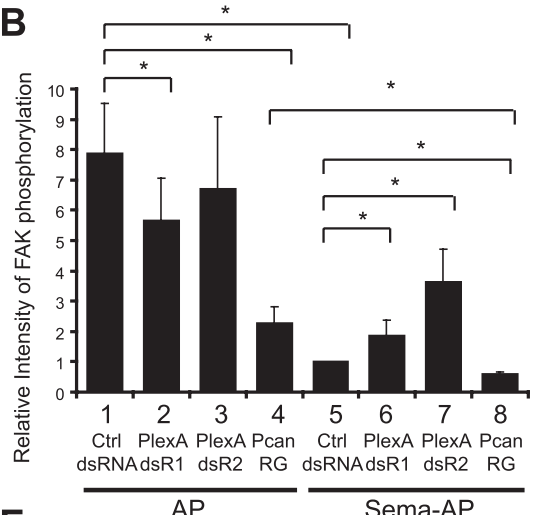

E

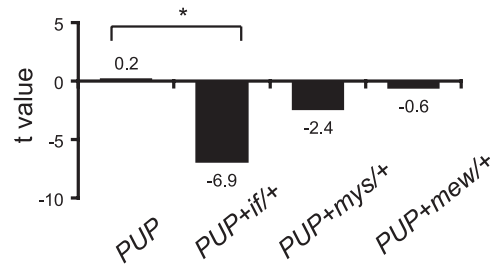

$\mathbf{F}$

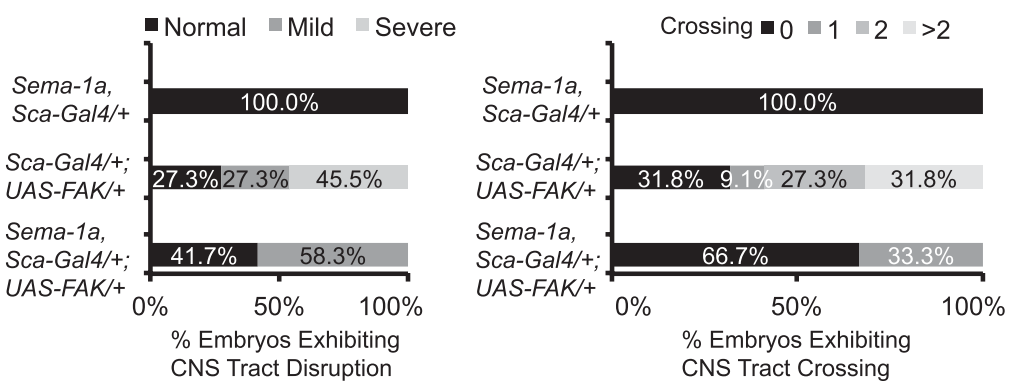

Figure 7. Perlecan enhances Sema-1a-mediated FAK dephosphorylation. (A, lanes 1,5) Thirty-minute treatment with 35 nM Sema-1aAP induces a decrease in endogenous phospho-FAK Y397 levels in S2R ${ }^{+}$cells. (Lanes 5-7) This decrease was significantly attenuated when the S2R ${ }^{+}$cells were pretreated with PlexA dsRNA. Perlecan overexpression alone decreases phospho-FAK level (lanes 1,4), and together with Sema-1a treatment, phospho-FAK levels are further reduced (lanes 5,8). (B) Quantification of multiple experiments shown in $A .\left(^{*}\right) P<0.01$. $\left(C\right.$, lanes 3,4) The reduction of phospho-FAK levels in $\mathrm{S}^{2} \mathrm{R}^{+}$cells overexpressing perlecan is attenuated when cells are pretreated with PlexA dsRNA. $(D)$ Quantification of multiple experiments shown in $\left.C .{ }^{\star}\right) P<0.01 .(E)$ if mutants significantly enhance the Sema-1a GOF phenotype. $\left({ }^{\star}\right) P<0.0001 .(F)$ Sema-1a and FAK GOF genetically interact. Removing one copy of Sema-1a suppresses FAK GOF CNS longitudinal tract phenotypes. The graph on the left shows the percentage of embryos exhibiting the lateral-most FasII ${ }^{+}$ longitudinal fascicle disruption broken down by severity, and the graph on the right presents the medial-most FasII ${ }^{+}$fascicle crossing broken down by the number of crossings.

for Sema-1a signaling. The lower level of enhancement by mys, as compared with if, may be due to redundancy with $\beta I n t-\nu$, a second Drosophila $\beta$ subunit (Nagaosa et al. 2011). Nevertheless, these results show that integrin and Sema-1a signaling act antagonistically in the context of CNS neuronal Sema-1a-mediated repulsive axon guidance.

\section{Discussion}

Our results provide support for the ability of ECM components to modulate axon guidance cue signaling. We show that transmembrane semaphorin signaling is modulated by the secreted HSPG perlecan, demonstrating that perlecan GOF and LOF mutants enhance and suppress, respectively, a robust Sema-1a GOF phenotype. We also observed that perlecan is required for endogenous repulsive Sema-1a-PlexA signaling in motor axons. Perlecan expression is elevated at motor axon defasciculation choice points, and neuronal expression of perlecan rescues guidance defects seen in perlecan LOF mutants. Our genetic analyses show that mutations in genes encoding other HSPGs, including syndecan and dally-like, do not affect Sema-1a neuronal functions. By assessing phospho-FAK levels in vitro, we found that perlecan can enhance Sema-1a-mediated down-regulation of phosphoFAK protein levels, and in vivo we observed that perlecan facilitates Sema-la antagonistic modulation of integrin signaling. Therefore, neuronal growth cone responses to the transmembrane guidance cue Sema-1a require facilitation by the ECM component perlecan, underscoring 
the importance of specific HSPG-guidance cue interactions in the assembly of neural circuits during development.

\section{Perlecan modulates transmembrane semaphorin signaling at motor axon defasciculation choice points}

The ECM includes collagens, glycoproteins, and proteoglycans that are produced by developing neurons and glia (Myers et al. 2011). Proteoglycans include versican, aggrecan, neurocan, and perlecan (Milev et al. 1998); however, with the exception of versican, our understanding of their in vivo axon guidance functions is incomplete (Dutt et al. 2011). In contrast, studies in mice using EXT mutants show that HSs are critical for slit-mediated midline axon guidance in vivo and also for Netrin/DCC signaling in spinal commissural axon guidance (Inatani et al. 2003; Matsumoto et al. 2007). In these EXT mutants, however, both transmembrane and GPI-linked HSPGs are affected, and it is not clear which HSPGs are important for these guidance events. Furthermore, the transmembrane semaphorin Sema5A can be modulated in vitro by both HSPGs and CSPGs (Kantor et al. 2004); however, the proteoglycans involved are also not known. Our present study provides insight into how a specific ECM proteoglycan modulates axonal responses to a well-characterized transmembrane guidance cue in vivo.

We found a strong correlation between perlecan localization and motor axon defasciculation choice points. This suggests that perlecan is localized at higher levels where increased Sema-1a-PlexA signaling is required. Our cell type-specific genetic rescue experiments, in combination with analysis of perlecan expression, suggest that perlecan is secreted by developing motor axons, which in turn require perlecan for choice point navigation. This likely occurs at specific interstitial locations along motor axon trajectories defined by glial cell ensheathment of motor nerves (Stork et al. 2008).

\section{HSPG specificity and the requirement for HS chains in Sema-1a signaling}

HSPGs in both invertebrates and vertebrates play key roles in axon guidance and synaptogenesis (Fox and Zinn 2005; Van Vactor et al. 2006). For example, in both Drosophila and $C$. elegans, syndecan regulates slit-Robo-mediated midline guidance events (Hu 2001; Johnson et al. 2004; Steigemann et al. 2004; Rhiner et al. 2005), and in Drosophila, dally-like can compensate for the loss of syndecan (Johnson et al. 2004). In contrast, during visual system assembly and synaptic development, there is only limited rescue of syndecan mutant defects with dallylike transgenes and vice versa; this suggests that dallylike and syndecan can serve distinct functions (Johnson et al. 2006). syndecan and dally-like mutants did not suppress or enhance the PUP Sema-1a GOF phenotype, whereas dally mutants were capable of enhancement. This likely reflects specificity conferred by perlecan and dally for critical interactions with Sema-1a signaling components.

Slit-Robo interactions are enhanced by HS, and HS is required for slit-mediated repulsion in vitro ( $\mathrm{Hu} 2001)$.
Furthermore, increased murine retinal axon guidance defects were observed when the dosage of Ext1 was lowered in a slit2 mutant (Inatani et al. 2003). In C. elegans, guidance cue signaling activity critically depends on different HS modifications (Bulow et al. 2008). To genetically assess perlecan HS contribution in Sema-1a signaling, we tested several HS biosynthetic mutants in the PUP Sema-1a GOF assay, and, surprisingly, both $s g l$ and $s f l$ mutants exhibited robust enhancement. One interpretation of these results is that HS chains on perlecan may interfere with the ability of the perlecan protein core to interact physically with Sema-1a or PlexA, and this would imply that the perlecan protein core is essential for facilitation of Sema-1a signaling. Alternatively, HS may serve autoinhibitory functions with respect to perlecan. Alternatively, HS chains on dally may be critical for dally-mediated signaling, since we observed that dally, sgl, and sfl mutants all enhance the Sema-1a GOF phenotype, and dally is a likely substrate of Sfl and Sgl (Lin and Perrimon 1999). These scenarios are not mutually exclusive. A key experiment is to mutate all of the potential HS sites on perlecan and test in vivo how this effects Sema-1a function.

Perlecan enhancement of Sema-1a-mediated FAK dephosphorylation in vitro highlights interactions between semaphorin and integrin signaling in vivo

Extending motor axons are tightly bundled through the action of cell adhesion molecules but also adhere the ECM as they navigate specific choice points. Our data support the idea that over most of a developing motor axon trajectory, lower perlecan levels lead to reduced activation of Sema-1a-PlexA signaling. However, when motor axons arrive at specific choice points, Sema-1aPlexA signaling is enhanced by perlecan, concomitantly down-regulating integrin-mediated adhesive interactions via FAK dephosphorylation and leading to motor axon defasciculation.

We found that mutations in integrin subunit genes significantly enhance the PUP Sema-1a GOF phenotype, thereby placing Sema-1a and integrin signaling in an antagonistic relationship, consistent with previous in vitro observations (Oinuma et al. 2006). Moreover, the robust FAK dephosphorylation that we observed following Sema-1a treatment in $\mathrm{S}_{2} \mathrm{R}^{+}$cells supports the idea that semaphorin and integrin signaling interact antagonistically. The mechanism underlying Sema-1a facilitation of FAK dephosphorylation is not known, and it remains to be determined whether this occurs through direct Sema-1a-PlexA interactions with integrin receptors or indirectly via plexin downstream signaling events. It is also possible that perlecan confers stronger binding affinity of Sema-1a to PlexA, thereby promoting the formation of an active semaphorin signaling complex at the expense of integrin signaling.

We examined Fak56 ${ }^{C G 1}$ mutant embryos and did not observe motor axon guidance defects (data not shown), consistent with the apparent normal development of Fak56 ${ }^{C G 1}$ mutants (Grabbe et al. 2004). One possibility is that FAK may not be essential owing to compensation 
by other protein tyrosine kinases. Nevertheless, the robust genetic interactions that we observed in CNS longitudinal tracts between Sema-1a LOF and FAK GOF suggest that FAK may function as an integrin effector such that Sema-1a and integrin signaling converge on FAK. Upon Sema-la signaling, FAK phosphorylation levels decrease, and this may inhibit integrin signaling. Our results also suggest that perlecan further inhibits integrin signaling by facilitating the Sema-1a signaling pathway.

In conclusion, we found that perlecan, a secreted ECM HSPG, is a critical component of Sema-la-mediated repulsive guidance in vivo. Furthermore, Sema-1a signaling apparently acts to antagonize integrin signaling. The regulated spatial localization of ECM components with the capacity to modulate specific guidance cue signaling pathways provides a robust mechanism for imparting localized responses to these cues, thereby facilitating the assembly of neural circuits during development.

\section{Materials and methods}

\section{Drosophila strains}

All mutant stocks have been previously described: $\operatorname{Plex} A^{D f(4) C 3}$ (Winberg et al. 1998), Pcan ${ }^{\text {null }}$ (Voigt et al. 2002), trol $^{8}$ (Park et al. 2003), EP1160 (Voigt et al. 2002), PlexB ${ }^{K G 00878}$ (Ayoob et al. 2006), dally ${ }^{80}$, dlp $p^{1}$ (Kirkpatrick et al. 2004), Sdc ${ }^{10608}$ (Johnson et al. 2004), sfl $1^{03844}, \operatorname{sgl}^{08310}, \operatorname{botv}^{510}$, sotv ${ }^{326}$ (Takei et al. 2004), and $t t v^{00681 b}$ (Bellaiche et al. 1998). elav-Gal4 was used for panneuronal labeling, 5053A-Gal4 (Swan et al. 2004) was used for muscle 12 labeling, repo-Gal4 for was used for glia labeling, twiGal4 was used for mesodermal expression labeling, and $\mathrm{Hb}$ 9Gal4 (Broihier and Skeath 2002) was used for neuronal labeling. For overexpression studies, we used UAS-Sema-1a (Yu et al. 1998), UAS-HA-PlexA (Winberg et al. 1998), UAS-Pcan-RG, UAS-Pcan-RD, UAS-dally (Bloomington Stock Center, 5397), UAS-CD8GFP (Bloomington Stock Center, 5130), UAS-FAK, and PlexA-BAC.

\section{Immunohistochemical analyses}

The antibodies used were anti-Sema-1a mAb (1:3000) (Yu et al. 1998), anti-perlecan (1:3000) (Friedrich et al. 2000), BP102 mAb (Seeger et al. 1993), anti-Fas II mAb 1D4 (Vactor et al. 1993), rabbit anti-GFP (1:1000; Molecular Probes), anti-Myc mAb 9E10 (1:1000; Sigma), anti-repo (1:10; Developmental Studies Hybridoma Bank, Iowa University), anti-wrapper (1:10) (Noordermeer et al. 1998), anti-MHC, HRP-conjugated goat anti-mouse and anti-rabbit IgG/M (1:500; Jackson Immunoresearch), Alexa488 or Alexa546-conjugated goat anti-mouse IgG, and Alexa647conjugated goat anti-rabbit IgG (1:500; Molecular Probes). Images were acquired using a Zeiss LSM 510 confocal microscope.

\section{Ligand production}

The extracellular portion of Sema-1a was cloned into the APtag5 vector (GenHunter) to generate a C-terminally AP-tagged fusion protein. The construct was then transfected into $293 \mathrm{~T}$ cells with Lipofectamine 2000 (0.5 $\mu \mathrm{g}$ DNA/ $1 \mu \mathrm{L}$ Lipofectamine). Three days after transfection, supernatants were collected and concentrated (Amicon Ultra 15). Ligand concentrations were measured by quantifying AP activity.

\section{dsRNA preparation}

To generate PlexA dsRNA, PlexA cDNA and primers containing T7 RNA polymerase-binding sites and gene-specific sequences to produce $\sim 500$-base-pair (bp) fragments containing $\mathrm{T} 7$ promoters at the $5^{\prime}$ ends were used. To minimize off-target effects, the "off-target sequence search tool" at the Drosophila RNAi Screening Center (DRSC) Web site was used. PCR products were purified and used as templates to produce RNA using the Roche T7 polymerase kit. RNA was NaOAc-precipitated, resuspended in water, and stored at $-80^{\circ} \mathrm{C}$. Control dsRNA was generated using the same method except using PlexB cDNA.

\section{$S 2 R^{+}$cell culture and treatment}

$\mathrm{S} \mathrm{R}^{+}$cells were grown in Schneider's medium (Invitrogen) with $10 \%$ heat-inactivated fetal bovine serum (FBS) at $25^{\circ} \mathrm{C}$ in culture flasks. Cells $\left(10^{5}\right)$ in $1 \mathrm{~mL}$ of medium were added to each well of a 12 -well plate, and $8 \mathrm{~h}$ later, dsRNA $(30 \mu \mathrm{g})$ was added to each well. The cells were then incubated for $24 \mathrm{~h}$ at $25^{\circ} \mathrm{C}$ before transfecting control or perlecan cDNA with Effectene reagent (Qiagen). Cells were grown for $1 \mathrm{~d}$ at $25^{\circ} \mathrm{C}$, and additional dsRNA $(30 \mu \mathrm{g})$ was added to each well. The next day, cells were washed briefly with PBS and treated with Sema-1a-AP or AP for 15 or 30 min depending on experimental purposes. Heparinase III $(0.2 \mu \mathrm{g} /$ $\mathrm{uL}$; Sigma) was used for 30 min before ligand treatment, when necessary. Cells were lysed in $100 \mu \mathrm{L}$ of lysis buffer $(0.5 \%$ NP-40, $50 \mathrm{nM}$ Tris $8.0,150 \mathrm{mM} \mathrm{NaCl}$, protease inhibitor, tyrosine phosphatase inhibitor) for $15 \mathrm{~min}$. Phospho-FAK rabbit $\mathrm{mAB}$ Y397 (1:500; Invitrogen) was used for Western blotting.

\section{Heparin-binding assay}

Heparin beads (Sigma H6508) were washed with Hanks' balanced salt solution (HBSS) and then blocked in HABH $(0.5 \mathrm{mg} / \mathrm{mL}$ BSA, $20 \mathrm{mM}$ HEPES at $\mathrm{pH} 7.0$ ) overnight at $4^{\circ} \mathrm{C}$. The beads were washed twice with $20 \mathrm{mM}$ HEPES (pH 7.0) and $150 \mathrm{mM} \mathrm{NaCl}$. $5 x m y c-S e m a l a$, PlexA-5xmyc $( \pm$ Semala $)$, or 5xmyc-dGIPC transfected cell lysates were incubated with the pretreated heparin beads overnight at $4^{\circ} \mathrm{C}$. The lysates were washed with $20 \mathrm{mM}$ HEPES (pH 7.0) and $150 \mathrm{mM} \mathrm{NaCl}$ seven times.

\section{Acknowledgments}

We thank T. Schupbach and S. Datta for perlecan mutants, S. Baumgartner for the perlecan antibody, D. Van Vactor for HSPG mutants and transgenic lines, T. Tabata for EXT mutants, $\mathrm{H}$. Nakato for HSME lines, R. Palmer for FAK mutants, D. Montell for the $F A K$ transgenic line, $\mathrm{Z}$. Wu for the PlexA-BAC line, E. Lee for statistical analyses, and the Bloomington Stock Center for fly stocks. We also thank Sangyun Jeong for critical comments on the manuscript. This work was supported by NIH R01 NS35165 to A.L.K. A.L.K. is an Investigator of the Howard Hughes Medical Institute.

\section{References}

Ayoob JC, Yu HH, Terman JR, Kolodkin AL. 2004. The Drosophila receptor guanylyl cyclase $\mathrm{Gyc} 76 \mathrm{C}$ is required for semaphorin-1a-plexin A-mediated axonal repulsion. J Neurosci 24: 6639-6649.

Ayoob JC, Terman JR, Kolodkin AL. 2006. Drosophila Plexin B is a Sema-2a receptor required for axon guidance. Development 133: 2125-2135.

Bashaw GJ, Klein R. 2010. Signaling from axon guidance receptors. Cold Spring Harb Perspect Biol 2: a001941. doi: 10.1101/cshperspect.a001941. 
Bellaiche Y, The I, Perrimon N. 1998. Tout-velu is a Drosophila homologue of the putative tumour suppressor EXT-1 and is needed for Hh diffusion. Nature 394: 85-88.

Bokel C, Brown NH. 2002. Integrins in development: Moving on, responding to, and sticking to the extracellular matrix. Dev Cell 3: 311-321.

Broihier HT, Skeath JB. 2002. Drosophila homeodomain protein $\mathrm{dHb} 9$ directs neuronal fate via crossrepressive and cellnonautonomous mechanisms. Neuron 35: 39-50.

Bulow HE, Tjoe N, Townley RA, Didiano D, van Kuppevelt TH, Hobert O. 2008. Extracellular sugar modifications provide instructive and cell-specific information for axon-guidance choices. Curr Biol 18: 1978-1985.

De Wit J, De Winter F, Klooster J, Verhaagen J. 2005. Semaphorin 3 A displays a punctate distribution on the surface of neuronal cells and interacts with proteoglycans in the extracellular matrix. Mol Cell Neurosci 29: 40-55.

Dutt S, Cassoly E, Dours-Zimmermann MT, Matasci M, Stoeckli ET, Zimmermann DR. 2011. Versican V0 and V1 direct the growth of peripheral axons in the developing chick hindlimb. J Neurosci 31: 5262-5270.

Fox AN, Zinn K. 2005. The heparan sulfate proteoglycan syndecan is an in vivo ligand for the Drosophila LAR receptor tyrosine phosphatase. Curr Biol 15: 1701-1711.

Friedrich MV, Schneider M, Timpl R, Baumgartner S. 2000. Perlecan domain V of Drosophila melanogaster. Sequence, recombinant analysis and tissue expression. Eur I Biochem 267: 3149-3159.

García-Alonso L, Fetter RD, Goodman CS. 1996. Genetic analysis of Laminin A in Drosophila: Extracellular matrix containing laminin A is required for ocellar axon pathfinding. Development 122: 2611-2621.

Grabbe C, Zervas CG, Hunter T, Brown NH, Palmer RH. 2004. Focal adhesion kinase is not required for integrin function or viability in Drosophila. Development 131: 5795-5805.

Hoang B, Chiba A. 1998. Genetic analysis on the role of integrin during axon guidance in Drosophila. J Neurosci 18: 7847-7855.

$\mathrm{Hu}$ H. 2001. Cell-surface heparan sulfate is involved in the repulsive guidance activities of Slit2 protein. Nat Neurosci 4: 695-701.

Inatani $\mathrm{M}$, Irie F, Plump AS, Tessier-Lavigne M, Yamaguchi Y. 2003. Mammalian brain morphogenesis and midline axon guidance require heparan sulfate. Science 302: 1044-1046.

Ivankovic-Dikic I, Gronroos E, Blaukat A, Barth BU, Dikic I. 2000. Pyk2 and FAK regulate neurite outgrowth induced by growth factors and integrins. Nat Cell Biol 2: 574-581.

Johnson KG, Ghose A, Epstein E, Lincecum J, O'Connor MB, Van Vactor D. 2004. Axonal heparan sulfate proteoglycans regulate the distribution and efficiency of the repellent slit during midline axon guidance. Curr Biol 14: 499-504.

Johnson KG, Tenney AP, Ghose A, Duckworth AM, Higashi ME, Parfitt K, Marcu O, Heslip TR, Marsh JL, Schwarz TL, et al. 2006. The HSPGs syndecan and dallylike bind the receptor phosphatase LAR and exert distinct effects on synaptic development. Neuron 49: 517-531.

Kamimura K, Maeda N, Nakato H. 2011. In vivo manipulation of heparan sulfate structure and its effect on Drosophila development. Glycobiology 21: 607-618.

Kantor DB, Chivatakarn O, Peer KL, Oster SF, Inatani M, Hansen MJ, Flanagan JG, Yamaguchi Y, Sretavan DW, Giger RJ, et al. 2004. Semaphorin $5 \mathrm{~A}$ is a bifunctional axon guidance cue regulated by heparan and chondroitin sulfate proteoglycans. Neuron 44: 961-975.

Katsuki T, Ailani D, Hiramoto M, Hiromi Y. 2009. Intra-axonal patterning: Intrinsic compartmentalization of the axonal membrane in Drosophila neurons. Neuron 64: 188-199.
Kirkpatrick CA, Dimitroff BD, Rawson JM, Selleck SB. 2004. Spatial regulation of Wingless morphogen distribution and signaling by Dally-like protein. Dev Cell 7: 513-523.

Kolodkin AL, Tessier-Lavigne M. 2011. Mechanisms and molecules of neuronal wiring: A primer. Cold Spring Harb Perspect Biol 3: a001727. doi: 10.1101/cshperspect.a001727.

Kolodziej PA, Timpe LC, Mitchell KJ, Fried SR, Goodman CS, Jan LY, Jan YN. 1996. frazzled encodes a Drosophila member of the DCC immunoglobulin subfamily and is required for CNS and motor axon guidance. Cell 87: 197-204.

Kornberg L, Earp HS, Parsons JT, Schaller M, Juliano RL. 1992. Cell adhesion or integrin clustering increases phosphorylation of a focal adhesion-associated tyrosine kinase. I Biol Chem 267: 23439-23442.

Landgraf M, Bossing T, Technau GM, Bate M. 1997. The origin, location, and projections of the embryonic abdominal motorneurons of Drosophila. J Neurosci 17: 9642-9655.

Lin X, Perrimon N. 1999. Dally cooperates with Drosophila Frizzled 2 to transduce Wingless signalling. Nature 400: 281284.

Matsumoto Y, Irie F, Inatani M, Tessier-Lavigne M, Yamaguchi Y. 2007. Netrin-1/DCC signaling in commissural axon guidance requires cell-autonomous expression of heparan sulfate. J Neurosci 27: 4342-4350.

Milev P, Maurel P, Chiba A, Mevissen M, Popp S, Yamaguchi Y, Margolis RK, Margolis RU. 1998. Differential regulation of expression of hyaluronan-binding proteoglycans in developing brain: Aggrecan, versican, neurocan, and brevican. Biochem Biophys Res Commun 247: 207-212.

Mitchell KJ, Doyle JL, Serafini T, Kennedy TE, Tessier-Lavigne M, Goodman CS, Dickson BJ. 1996. Genetic analysis of Netrin genes in Drosophila: Netrins guide CNS commissural axons and peripheral motor axons. Neuron 17: 203-215.

Myers JP, Santiago-Medina M, Gomez TM. 2011. Regulation of axonal outgrowth and pathfinding by integrin-ECM interactions. Dev Neurobiol 71: 901-923.

Nagaosa K, Okada R, Nonaka S, Takeuchi K, Fujita Y, Miyasaka T, Manaka J, Ando I, Nakanishi Y. 2011. Integrin $\beta v$-mediated phagocytosis of apoptotic cells in Drosophila embryos. J Biol Chem 286: 25770-25777.

Noordermeer JN, Kopczynski CC, Fetter RD, Bland KS, Chen WY, Goodman CS. 1998. Wrapper, a novel member of the Ig superfamily, is expressed by midline glia and is required for them to ensheath commissural axons in Drosophila. Neuron 21: 991-1001.

Odden JP, Holbrook S, Doe CQ. 2002. Drosophila HB9 is expressed in a subset of motoneurons and interneurons, where it regulates gene expression and axon pathfinding. I Neurosci 22: 9143-9149.

Oinuma I, Katoh H, Negishi M. 2006. Semaphorin 4D/PlexinB1-mediated R-Ras GAP activity inhibits cell migration by regulating $\beta 1$ integrin activity. J Cell Biol 173: 601-613.

Park Y, Rangel C, Reynolds MM, Caldwell MC, Johns M, Nayak M, Welsh CJ, McDermott S, Datta S. 2003. Drosophila perlecan modulates FGF and hedgehog signals to activate neural stem cell division. Dev Biol 253: 247-257.

Pasterkamp RJ, Peschon JJ, Spriggs MK, Kolodkin AL. 2003. Semaphorin 7A promotes axon outgrowth through integrins and MAPKs. Nature 424: 398-405.

Raper J, Mason C. 2010. Cellular strategies of axonal pathfinding. Cold Spring Harb Perspect Biol 2: a001933. doi: 10.1101/ cshperspect.a001933.

Rawson JM, Dimitroff B, Johnson KG, Ge X, Van Vactor D, Selleck SB. 2005. The heparan sulfate proteoglycans Dallylike and Syndecan have distinct functions in axon guidance and visual-system assembly in Drosophila. Curr Biol 15: 833-838. 
Rhiner C, Gysi S, Frohli E, Hengartner MO, Hajnal A. 2005. Syndecan regulates cell migration and axon guidance in $C$. elegans. Development 132: 4621-4633.

Ritzenthaler S, Suzuki E, Chiba A. 2000. Postsynaptic filopodia in muscle cells interact with innervating motoneuron axons. Nat Neurosci 3: 1012-1017.

Roberts A, Taylor JS. 1982. A scanning electron microscope study of the development of a peripheral sensory neurite network. J Embryol Exp Morphol 69: 237-250.

Robles E, Gomez TM. 2006. Focal adhesion kinase signaling at sites of integrin-mediated adhesion controls axon pathfinding. Nat Neurosci 9: 1274-1283.

Schlomann U, Schwamborn JC, Muller M, Fassler R, Puschel AW. 2009. The stimulation of dendrite growth by Sema3A requires integrin engagement and focal adhesion kinase. J Cell Sci 122: 2034-2042.

Seeger M, Tear G, Ferres-Marco D, Goodman CS. 1993. Mutations affecting growth cone guidance in Drosophila: Genes necessary for guidance toward or away from the midline. Neuron 10: 409-426.

Serini G, Valdembri D, Zanivan S, Morterra G, Burkhardt C, Caccavari F, Zammataro L, Primo L, Tamagnone L, Logan $\mathrm{M}$, et al. 2003. Class 3 semaphorins control vascular morphogenesis by inhibiting integrin function. Nature 424: 391-397.

Steigemann P, Molitor A, Fellert S, Jackle H, Vorbruggen G. 2004. Heparan sulfate proteoglycan syndecan promotes axonal and myotube guidance by slit/robo signaling. Curr Biol 14: 225-230.

Stork T, Engelen D, Krudewig A, Silies M, Bainton RJ, Klambt C. 2008. Organization and function of the blood-brain barrier in Drosophila. J Neurosci 28: 587-597.

Swan LE, Wichmann C, Prange U, Schmid A, Schmidt M, Schwarz T, Ponimaskin E, Madeo F, Vorbruggen G, Sigrist SJ. 2004. A glutamate receptor-interacting protein homolog organizes muscle guidance in Drosophila. Genes Dev 18: 223-237.

Takei Y, Ozawa Y, Sato M, Watanabe A, Tabata T. 2004. Three Drosophila EXT genes shape morphogen gradients through synthesis of heparan sulfate proteoglycans. Development 131: $73-82$.

Tessier-Lavigne M, Goodman CS. 1996. The molecular biology of axon guidance. Science 274: 1123-1133.

Tran TS, Kolodkin AL, Bharadwaj R. 2007. Semaphorin regulation of cellular morphology. Annu Rev Cell Dev Biol 23: 263-292.

Vactor DV, Sink H, Fambrough D, Tsoo R, Goodman CS. 1993. Genes that control neuromuscular specificity in Drosophila. Cell 73: 1137-1153.

Van Vactor D, Wall DP, Johnson KG. 2006. Heparan sulfate proteoglycans and the emergence of neuronal connectivity. Curr Opin Neurobiol 16: 40-51.

Voigt A, Pflanz R, Schafer U, Jackle H. 2002. Perlecan participates in proliferation activation of quiescent Drosophila neuroblasts. Dev Dyn 224: 403-412.

Winberg ML, Noordermeer JN, Tamagnone L, Comoglio PM, Spriggs MK, Tessier-Lavigne M, Goodman CS. 1998. Plexin A is a neuronal semaphorin receptor that controls axon guidance. Cell 95: 903-916.

Winckler B, Mellman I. 2010. Trafficking guidance receptors. Cold Spring Harb Perspect Biol 2: a001826. doi: 10.1101/ cshperspect.a001826.

Wu Z, Sweeney LB, Ayoob JC, Chak K, Andreone BJ, Ohyama T, Kerr R, Luo L, Zlatic M, Kolodkin AL. 2011. A combinatorial semaphorin code instructs the initial steps of sensory circuit assembly in the Drosophila CNS. Neuron 70: 281-298.
Yu HH, Araj HH, Ralls SA, Kolodkin AL. 1998. The transmembrane semaphorin Sema I is required in Drosophila for embryonic motor and CNS axon guidance. Neuron 20: 207220. 


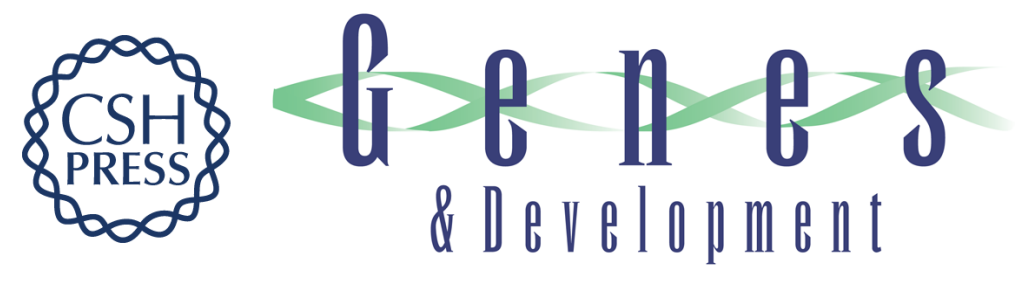

\section{The extracellular matrix proteoglycan perlecan facilitates transmembrane semaphorin-mediated repulsive guidance}

Joong Youn Cho, Kayam Chak, Benjamin J. Andreone, et al.

Genes Dev. 2012, 26:

Access the most recent version at doi:10.1101/gad.193136.112

Supplemental http://genesdev.cshlp.org/content/suppl/2012/09/26/26.19.2222.DC1
Material

References This article cites 60 articles, 23 of which can be accessed free at:

http://genesdev.cshlp.org/content/26/19/2222.full.html\#ref-list-1

License

Email Alerting Receive free email alerts when new articles cite this article - sign up in the box at the top

Service

right corner of the article or click here.

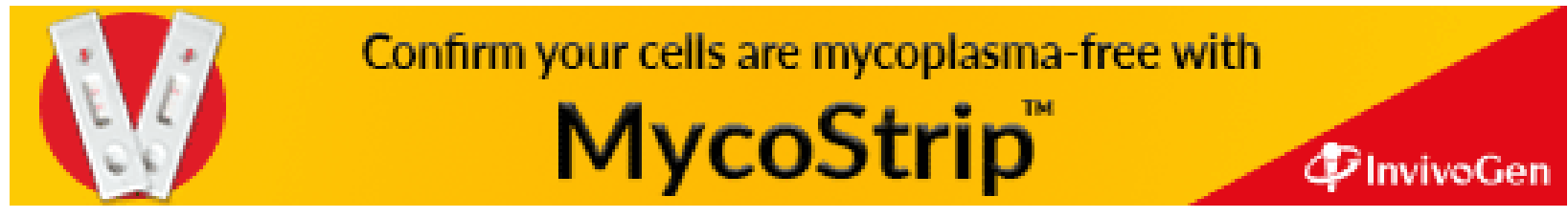

